\title{
More than Half of Patients with Schizophrenia Are Receiving Polypharmacy and Co-Prescription of Anxiolytics in Pakistan -Findings from Research on Asian Prescription Pattern in 2016
}

\author{
Mazhar Malik1, Usman Ghani1', Nariman Awais'1, Nargis Munir1, Afzal Javed², \\ Munir Hamirani' ${ }^{3}$, Nasar Sayeed Kahn", Shu-Yu Yang5, Liang-Yu Chen6, Shih-Ku Lin7, \\ Norman Sartorius ${ }^{8}$, Chay-Hoon Tan ${ }^{9}$, Mian-Yoon Chong10, Masaki Shinfuku' ${ }^{11}$, \\ Sandeep Grover ${ }^{12}$, Winston W. Shen ${ }^{13}$, Naotaka Shinfuku ${ }^{14 *}$ \\ ${ }^{1}$ Department of Psychiatry and Behavioral Sciences, Rawal Institute of Health Sciences, Islamabad, Pakistan \\ ${ }^{2}$ Pakistan Psychiatric Research Center, Fountain House, Lahore, Pakistan \\ ${ }^{3}$ Department of Psychiatry, Abbasi Shaheed Hospital, Karachi, Pakistan \\ ${ }^{4}$ Department of Psychiatry, Services Hospital, Lahore, Pakistan \\ ${ }^{5}$ Taipei City Hospital and Psychiatric Center, Taiwan \\ ${ }^{6}$ Institute of Epidemiology and Preventive Medicine, National Taiwan University, Taiwan \\ ${ }^{7}$ Department of Psychiatry, School of Medicine, Taipei Medical University, Taiwan \\ ${ }^{8}$ Association for the Improvement of Mental Health Programs, Geneva, Switzerland \\ ${ }^{9}$ National University of Singapore, Singapore \\ ${ }^{10}$ Chiayi Chang Gung Memorial Hospital and School of Medicine, Chang Gung University, Taiwan \\ ${ }^{11}$ Department of Psychiatry, Tokyo Medical Center, Tokyo, Japan \\ ${ }^{12}$ Department of Psychiatry, Post Graduate Institute of Medical Education and Research (PIGMER), Chandigah, India \\ ${ }^{13}$ Departments of Psychiatry, Medical Center and College of Medicine, Taipei Medical University, Taiwan \\ ${ }^{14}$ Kobe University School of Medicine, Kobe, Japan \\ Email: *shinfukunaotaka@gmail.com
}

How to cite this paper: Malik, M., Ghani, U., Awais, N., Munir, N., Javed, A., Hamirani, M., Kahn, N.S., Yang, S.-Y., Chen, L.-Y., Lin, S.-K., Sartorius, N., Tan, C.-H., Chong, M.-Y., Shinfuku, M., Grover, S., Shen, W.W. and Shinfuku, N. (2018) More than Half of Patients with Schizophrenia Are Receiving Polypharmacy and Co-Prescription of Anxiolytics in Pakistan -Findings from Research on Asian Prescription Pattern in 2016. Open Journal of Psychiatry, 8, 199-211.

https://doi.org/10.4236/ojpsych.2018.83018

\begin{abstract}
Objective: Little is known about the prescription pattern of psychotropic drugs for patients with schizophrenia in Pakistan. The purpose of this study was to evaluate the characteristic features of psychotropic drug prescriptions for patients with schizophrenia in Pakistan. Methods: Three centers in Pakistan participated in a large scale collaborative study known as Research on Asian Prescription Pattern (REAP). The 2016 REAP survey included centers from 15 countries in Asia and used a unified research protocol. The design of the study was quantitative and of descriptive epidemiology. Analysis was made on the data collected from three centers i.e., Lahore, Karachi and Isla-
\end{abstract}


Received: January 2, 2018

Accepted: June 5, 2018

Published: June 8, 2018

Copyright (c) 2018 by authors and Scientific Research Publishing Inc. This work is licensed under the Creative Commons Attribution International

License (CC BY 4.0).

http://creativecommons.org/licenses/by/4.0/ mabad. The data collected in Pakistan were compared with those from other Asian countries. The details of REAP were presented on the homepage of REAP (http://www.REAP.Asia). Results: From Pakistan, 298 patients were included. Patients with schizophrenia in Pakistan received higher rate of antipsychotic polypharmacy and a higher rate of co-prescription of mood stabilizers and anxiolytics when compared with participants from other South Asian countries (India and Bangladesh). Conclusion: The main findings of the study were that a majority of the patients were prescribed antipsychotic polypharmacy drugs in Pakistan. Insufficient training on the use of psychotropic drugs and a lack of treatment guideline are considered to be the major contributing factors. Further education and training on the proper use of psychotropic drugs are recommended to psychiatrists in Pakistan. The guidelines on pharmacotherapy for patients with schizophrenia should also be developed and promoted in Pakistan.

\section{Keywords}

Pakistan, Psychotropic Drugs, Polypharmacy, REAP, Schizophrenia

\section{Introduction}

The Research on East Asian Psychotropic Prescription Pattern (REAP) is the largest and the longest standing international collaborative research project in Asia. REAP has been a large scale survey of prescription patterns of patients with schizophrenia in different countries in Asia since 2001 [1], [2].

Before centers in Pakistan participated in the REAP survey for the first time in 2016, little was known about the prescription pattern of psychotropic drugs in Pakistan. This study is the first large scale survey on psychotropic prescription pattern based on a unified research protocol.

Mental health services in Pakistan have been shown by the World Health Organization Assessment Instrument for Mental Health Systems (WHO-AIMS). The number of psychiatric resources is very limited in Pakistan for the huge population of around 180 million. The psychiatric beds per 10,000 population is around 0.12 . Three centers which participated in the survey were leading psychiatric institutions for training and research. Patients suffering from schizophrenia are most commonly treated at inpatient- and community-based psychiatric settings. The length of stay in the hospitals is ranged from 17 to 50 days. Psychotropic drugs are mostly widely available at mental hospitals, followed by inpatient units, and then outpatient mental health facilities. The total number of mental health professionals includes 342 psychiatrists, 478 psychologists, 3145 social workers, 22 occupational therapists, and a large number of other health or mental health workers. More than 25,000 medical doctors in other fields and more than 13,000 nurses also support mental health services in Pakistan [3]. The distribution between urban and rural areas is disproportionate. The number of psychiatrists and nurses available in or around the large cities is greater than that 
in rural areas in the entire country. Public education and awareness campaigns on mental health are governed by a coordinating body with the help of government agencies [3]

The limited mental health resources are used mostly to provide services to patients with schizophrenia with gross behavior manifestations. The median age of the population is below 25 years, and this results in the big number of high risk population for schizophrenia in Pakistan. Schizophrenia is a disorder distinguished by a mixed nature, complex history, symptoms and types that can be identified on the basis of its course, outcome and its response to treatment. The use of psychotropic drug is the mainstream of treatment for persons with schizophrenia in Pakistan [4].

The treatment of schizophrenia has always been a big challenge despite tremendous pharmacologic progress [5]. The antipsychotic drugs are renowned to treat symptoms of schizophrenia and have been helpful in averting relapse of the illness for many years [6].

Polypharmacy is defined as concurrent use of multiple medications prescribed by physicians. The prescription of two or more antipsychotic drugs has also been described as polypharmacy [7]. Antipsychotic polypharmacy (APP) has been the usual mode of treatment for patients with schizophrenia spectrum disorders, despite scanty evidence of their beneficial effects.

Besides antipsychotics, anxiolytics widely known for reducing anxiety and for their sleep-inducing properties, are also used for their sedative, anticonvulsant and muscle relaxing properties for treating a wide spectrum of psychiatric and non-psychiatric illnesses [8], [9].

About $40 \%$ of psychiatric patients with co-morbidities in Karachi, Pakistan, have been prescribed anxiolytics by their general practitioners [10].

Furthermore, antidepressants, mood stabilizers and anti-Parkinsonian drugs have been prescribed by the psychiatrists all worldwide. The practice of co-prescription is widespread in Southeast Asian countries for multiple reasons, but the practice is used primarily to curtail the difficult symptoms that accompany chronic psychiatric illnesses.

The objectives of this study were to examine the characteristic features of psychotropic drug prescriptions for patients with schizophrenia in Pakistan, and to suggest the ways of improving the dispensation of psychotropic prescription.

\section{Methods}

The design of this study was quantitative through use of a descriptive epidemiologic survey. Data were collected based on a unified protocol by participating psychiatrists at centers in the survey. Three centers in Lahore, Karachi and Islamabad provided the data. Inpatients and outpatients with schizophrenia were recruited.

The inclusion criteria was that the patients had been diagnosed with schizophrenia according to the Diagnostic and Statistical Manual for Mental Disorders (DSM-5) or the International Classification of Diseases (ICD-10). (DSM-5) 
developed by American Psychiatric Association (APA) and (ICD-10) developed by WHO are commonly used in Asia. Individuals who refused to participate in the study, or who had serious physical illnesses were excluded.

Study protocol was approved by the Medical Ethics Committee of the Rawal Institute of Health Sciences Islamabad, Pakistan, with the requirement that signed informed consents were obtained from the participants. We collected the data using a questionnaire that included demographic details, psychiatric symptoms, psychotropic medications, comorbidities, physical conditions, and adverse drug reactions. A website-based recording system was used to send collected data to a computer center at Taipei Municipal Hospital. Compilation and analysis of data were carried out by a computer team in Taipei, Taiwan. The study results of the statistical analysis were transferred to all participating centers, including those in Pakistan. This paper analyzed the data collected from centers in Pakistan and compared them with respective data received from other countries and areas.

\section{Results}

The number of patients, psychiatrists and hospitals who participated in the REAP survey in 2016 from Pakistan and other 14 countries and areas are presented (Table 1). The survey was conducted for three months from April to June 2016.

Across the three centers, 298 patients were included: Lahore 247, Karachi 13, and Islamabad 38. The majority of cases were collected from Lahore (86.6\%). The mean age of the patients was 36.9 years, 168 (56.4\%) of the subjects were males and $130(43.6 \%)$ were females. Disease duration from the onset was less than 5 years for 112 patients (38.1\%). On the other hand, 119 patients (40.3\%) had a history of illness for more than 10 years. The collected data include both acute and chronic patients. Of these 298 patients, 156 (52.3\%) were outpatients and $142(47.7 \%)$ were inpatients (Table 2).

The proportion of the patients using typical (first-generation) antipsychotics (FGAs), atypical (second-generation) antipsychotics (SGAs) and on anti-psychotic polypharmacy (APP) is presented in Table 3. Out of 298 patients, 288 (96.6\%) patients were prescribed anti-psychotics. Seventy two patients (24.2\%) were prescribed long-acting antipsychotics (LAAs). Ninety-seven patients (32.6\%) received the FGAs, and 243 patients (81.2\%) received SGAs. A considerable number of patients received both FGAs and SGAs. LAAs were also used in combination with FGAs and SGAs.

There were 46 patients (15.3\%) receiving FGA monotherapy, 94 (31.6\%) receiving SGA monotherapy and 151 (51.7\%) receiving both FGA and SGA.

The list of the most commonly prescribed antipsychotic drugs in Pakistan was reviewed for 288 patients who were prescribed antipsychotic drugs. Of all the patients, 186 patients $(62.4 \%)$ were on risperidone which was the most commonly prescribed antipsychotic drug followed by $79(26.5 \%)$ patients on haloperidol, 55 
Table 1. REAP-AP4 study subjects and sites $(\mathrm{n}=3744)$.

\begin{tabular}{|c|c|c|c|c|c|}
\hline & \multicolumn{3}{|c|}{ Patients } & \multirow{2}{*}{ Psychiatrists } & \multirow{2}{*}{ Hospitals } \\
\hline & ALL & Inpatient & Outpatient & & \\
\hline Bangladesh & 99 & 48 & 51 & 2 & 1 \\
\hline China & 160 & 146 & 14 & 24 & 2 \\
\hline Hong Kong & 31 & 31 & 0 & 11 & 1 \\
\hline India & 479 & 150 & 329 & 20 & 5 \\
\hline Indonesia & 581 & 289 & 292 & 61 & 9 \\
\hline Japan & 229 & 129 & 100 & 44 & 8 \\
\hline Korea & 131 & 6 & 125 & 14 & 3 \\
\hline Malaysia & 305 & 103 & 202 & 21 & 10 \\
\hline Myanmar & 164 & 90 & 74 & 23 & 5 \\
\hline Pakistan & 298 & 142 & 156 & 32 & 3 \\
\hline Singapore & 171 & 128 & 43 & 10 & 2 \\
\hline Sri Lanka & 97 & 50 & 47 & 11 & 3 \\
\hline Taiwan & 403 & 224 & 179 & 17 & 5 \\
\hline Thailand & 322 & 140 & 182 & 50 & 4 \\
\hline Vietnam & 274 & 274 & 0 & 95 & 10 \\
\hline Total & 3744 & 1950 & 1794 & 435 & 71 \\
\hline
\end{tabular}

Table 2. Demographic details of patients from three centers of Pakistan $(n=298)$.

\begin{tabular}{|c|c|c|c|c|}
\hline \multirow{2}{*}{ In n (\%) } & Total & Lahore & Karachi & Islamabad \\
\hline & 298 & $247(86.6)$ & $13(04.3)$ & $38(13.0)$ \\
\hline Age (20 - 65 yrs), mean (SD) & $36.9(11.9)$ & $36.6(11.9)$ & $41.2(11.7)$ & $37.0(11.9)$ \\
\hline \multicolumn{5}{|l|}{ Gender, n (\%) } \\
\hline Male & $168(56.4)$ & $138(56.1)$ & $8(61.5)$ & $22(56.4)$ \\
\hline Female & $130(43.6)$ & $108(43.9)$ & $5(38.5)$ & $17(43.6)$ \\
\hline \multicolumn{5}{|l|}{ Duration from the onset to date } \\
\hline Less than 1year & $41(14.0)$ & $34(14.1)$ & 0 & $7(17.9)$ \\
\hline 1 year - Less than 5 years & $71(24.1)$ & $63(25.9)$ & 0 & $8(20.5)$ \\
\hline 5 year - Less than 10 years & $64(21.7)$ & $47(19.3)$ & $5(38.5)$ & $12(30.8)$ \\
\hline 10 year - Less than 20 years & $73(24.7)$ & $58(23.9)$ & $5(38.5)$ & $10(25.6)$ \\
\hline More than 20 years & $46(15.6)$ & $41(16.9)$ & $3(23.1)$ & $2(5.1)$ \\
\hline \multicolumn{5}{|l|}{ Treatment setting, $\mathrm{n}(\%)$} \\
\hline Outpatient & $156(52.3)$ & $108(43.9)$ & $11(84.6)$ & $37(94.9)$ \\
\hline Inpatient & $142(47.7)$ & $138(56.1)$ & $2(15.4)$ & $2(5.1)$ \\
\hline \multicolumn{5}{|l|}{ Course of illness for the past year, $\mathrm{n}(\%)$} \\
\hline Remission & $112(37.6)$ & $78(31.7)$ & $3(23.1)$ & $31(79.5)$ \\
\hline Continuing presence of symptoms & $187(62.8)$ & $170(69.1)$ & $10(76.9)$ & $7(17.9)$ \\
\hline
\end{tabular}


Table 3. Antipsychotic drug use and Polypharmacy from three centers of Pakistan $(\mathrm{n}=$ 298).

\begin{tabular}{ccccc}
\hline \multirow{2}{*}{ In n (\%) } & Total & Lahore & Karac & Islamabad \\
\cline { 2 - 5 } & 298 & $247(86.6)$ & $13(04.3)$ & $38(13.0)$ \\
\hline Antipsychotic used, n (\%) & $288(96.6)$ & $236(95.9)$ & $13(100.0)$ & $39(100.0)$ \\
Long-acting & $72(24.2)$ & $54(22.0)$ & $4(30.8)$ & $14(35.9)$ \\
First-generation & $97(32.6)$ & $87(35.4)$ & $2(15.4)$ & $8(20.5)$ \\
$\begin{array}{c}\text { Second-generation } \\
\text { Number of antipsychotic used, } \\
\text { n (\%) }\end{array}$ & $243(81.5)$ & $194(78.9)$ & $12(92.3)$ & $37(94.9)$ \\
More than one & $154(51.7)$ & $130(52.8)$ & $5(38.5)$ & $19(48.7)$ \\
Concurrent psychotropic drug \\
used, n (\%)
\end{tabular}

(18.5\%) on quetiapine, 38 (12.8\%) on flupenazine decanoate, $33(11.1 \%)$ patients on clozapine, $20(6.7 \%)$, on zulcopenthixol decanoate $18(6.0 \%)$. on olanzapine, and $18(6.0 \%)$ on aripiprazole (Table 4$)$. This shows almost all commonly prescribed psychotropic drugs worldwide are also available in Pakistan. The list is similar to that of other Asian countries. The data form REAP survey shows that most FGAs and SGAs are available even in countries with limited resources in Asia [1], [2].

Antipsychotic polypharmacy and combined medication is Pakistan was reviewed along with four other countries who participated in the survey. They are Bangladesh, India, Japan and Singapore. Bangladesh and India have a similar mental health service delivery system. Japan and Singapore are rich in psychiatric manpower and facilities.

The rate of polypharmacy in Pakistan (51.7\%) was higher than in India (25.7\%), and Bangladesh (45.5\%) but lower than in Singapore (52.6\%) and Japan (55.0\%). The mean number of antipsychotics in Pakistan was 1.6 (SD 0.8) and was higher than that of India (1.3) and Bangladesh (1.5). The rate was the same as that in Singapore (1.6) but lower than Japan (1.8).

The dosage of antipsychotic drugs as measured by the prescribed daily dose (PDD)/defined daily dose (DDD) was also calculated. The sum of each antipsychotic's PDD/DDD in Pakistan (1.79) was higher than in India (1.33) and Singapore 81.47) but lower than Bangladesh (2.17) and Japan (2.29). These figures shows schizophrenic patients in Pakistan receive higher dose of antipsychotic 
Table 4. Frequently used antipsychotic drugs in Pakistan.

\begin{tabular}{|c|c|c|c|}
\hline Class of Drugs & Drug Name & N 298 & $\%$ \\
\hline \multicolumn{4}{|l|}{$\begin{array}{l}\text { First-generation } \\
\text { antipsychotics }\end{array}$} \\
\hline & Haloperidol $^{2}$ & 79 & $(26.5 \%)$ \\
\hline & Trifluoperazine ${ }^{7}$ & 17 & $5.7 \%$ \\
\hline & Clopenthixol $^{10}$ & 1 & $0.3 \%$ \\
\hline & Chlorpromazine $^{10}$ & 1 & $0.3 \%$ \\
\hline \multicolumn{4}{|l|}{$\begin{array}{l}\text { Second-generation } \\
\text { antipsychotics }\end{array}$} \\
\hline & Risperidone $^{1}$ & 186 & $62.4 \%$ \\
\hline & Quetiapine $^{3}$ & 55 & $18.5 \%$ \\
\hline & Clozapine $^{4}$ & 33 & $11.1 \%$ \\
\hline & Aripiprazole $^{6}$ & 18 & $6.0 \%$ \\
\hline & Olanzapine $^{6}$ & 18 & $6.0 \%$ \\
\hline & Amisulpride $^{9}$ & 2 & $0.7 \%$ \\
\hline & Ziprasidone $^{9}$ & 2 & $0.7 \%$ \\
\hline \multirow[t]{4}{*}{ Long-actingantipsychotics } & Fluphenazine decanoate ${ }^{4}$ & 38 & $12.8 \%$ \\
\hline & Zuclopenthixol decanoate ${ }^{5}$ & 20 & $6.7 \%$ \\
\hline & Flupentixol decanoate ${ }^{8}$ & 8 & $2.7 \%$ \\
\hline & Haloperidol decanoate ${ }^{8}$ & 8 & $2.7 \%$ \\
\hline
\end{tabular}

drugs when compared to India and Bangladesh which share similar socio-cultural and developmental background and mental health service systems.

The use of co-prescription of other psychotropic drugs along with antipsychotics to schizophrenic patients was also analyzed. . We found that mood stabilizer was prescribed for 77 patients (25.8\%). The percentage in Pakistan was much higher than that of Singapore (17.5\%), India (7.1\%) and Bangladesh (1.0\%) but lower than Japan, where more than $30 \% \mathrm{v}$ of patients with schizophrenia are co-prescribed mood stabilizers.

Antidepressants were co-prescribed to 56 patients (18.8\%) in Pakistan. The rate was higher than that in Bangladesh (0\%), Japan (2.6\%), and India (15.2\%) but lower than that in Singapore (23.4\%). The rate of co-prescription of anxiolytic was the highest in Pakistan. Anxiolytic was prescribed to 166 (57.5\%) of the 298 schizophrenic patients. Clonazepam prescribed to 116 patients (38.9\%), and lorazepam prescribed to 36 patients (12.1\%) are frequently co-prescribed.

Hypnotics were prescribed to 21 patients (7\%). This rate was higher than in India (1.5\%), Bangladesh (4.0\%) and Singapore (5.8\%) However, hypnotics was prescribed to more than $60 \%$ of patients with schizophrenia in Japan.

Antiparkinsonian drugs are also frequently co-prescribed for patients with schizophrenia in Pakistan. In this study, 207 patients (65.9\%) were prescribed 
such drugs. The rate was lower than that in Bangladesh (87.9\%), but higher than that in Singapore (40.6\%), Japan (39.3\%) and India (37.6\%).

\section{Discussion}

Pakistan was once a British colony, so psychiatry is strongly influenced by British mental health practice guidelines. As mentioned in the introduction, mental health resources in Pakistan are limited. The three centers that participated in the survey are the major psychiatric centers in the country.

The rate of polypharmacy of antipsychotic drugs in Pakistan in the current REAP study was found to be high (51.7\%) as compared to that in India $(25.7 \%)$ and that in Bangladesh (45.5\%) (Table 5). As a rule, polypharmacy is more prevalent in rich countries such as Japan (55.0\%) and Singapore (52.6\%). Before the survey was undertaken, it was not expected that more than half of the schizophrenic patients in Pakistan would have psychotropic polypharmacy.

Our survey showed that SGAs are most commonly used for the treatment of patients with schizophrenia. Risperidone was the most frequently prescribed (62.4\%). Quetiapine and clozapine were prescribed for more than $10 \%$ of the patients. The prescription of olanzapine was low due to its high cost and side effects (Table 4). Among FGAs, haloperidol was the most commonly prescribed (26.7\%). Our study and many other Asian studies indicated that the rate of polypharmacy in Asia is high [11]. Polypharmacy has become a common clinical practice for many psychiatric conditions and has become the usual method of treatment for mental health care all over the world. [12], [13]. Another study conducted in Pakistan for elderly people with schizophrenia reported that a half of the patients (50\%) were treated with SGAs, 14\% of patients were treated with FGAs, and $22 \%$ of them were treated with both FGAs and SGAs. Fourteen percent of the patients did not receive any antipsychotic drugs [14]. These findings are consistent with our study. In Pakistan, polypharmacy is more commonly observed in patients with an ICD-10 diagnosis of schizophrenia, schizotypal, or delusional disorders [15].

Polypharmacy is multi-factorial. Polypharmacy often occurs when the illness is refractory to primary treatment, but also when the doctors find it difficult to manage side effects or when a diagnostic dilemma exists. Sub-therapeutic doses of a drug may lead to an insufficient response, after which more medications are added. Poor compliance is an important factor contributing to polypharmacy. Further, many patients tend to self-medicate themselves in a country where drugs can be easily prescribed over the counter. Physicians sometimes focus on drugs as a primary solution, thus placing less focus on the usefulness of non-pharmacological interventions.

Doctors in Pakistan may believe that a single medication is not effective enough in treating the many symptoms of patients with schizophrenia. Polypharmacy is often caused by doctor's belief that multiple drugs are necessary to treat individual symptoms, co-morbid illnesses, unremitting symptoms, and extrapyramidal effects. This is often incorrect. 
Table 5. Antipsychotic polypharmacy and combined medications in five countries in Asia.

\begin{tabular}{ccccccc}
\hline & $\mathrm{N}(\%)$ & Bangladesh & India & Pakistan & Japan & Singapore \\
\cline { 2 - 7 } & $\mathbf{n}=$ & 99 & 479 & 298 & 229 & 171 \\
\hline $\begin{array}{c}\text { Polypharmacy } \\
\text { (More than 1 }\end{array}$ & $\mathrm{N}$ & 45 & 123 & 154 & 126 & 90 \\
antipsychotic) & $\%$ & $(45.5)$ & $(25.7)$ & $(51.7)$ & $(55.0)$ & $(52.6)$ \\
Mean number of & mean & 1.5 & 1.3 & 1.6 & 1.8 & 1.6 \\
antipsychotic used & $\mathrm{SD}$ & 0.5 & 0.5 & 0.8 & 0.9 & 0.6 \\
Sum of each & & & & & & \\
antipsychotic's & mean & 2.17 & 1.33 & 1.79 & 2.29 & 1.47 \\
PDD/DDD & $\mathrm{SD}$ & 1.01 & 0.92 & 1.47 & 1.79 & 0.99 \\
& & & & & & \\
Mood stabilizer used & $\mathrm{N}$ & 1 & 34 & 77 & 69 & 30 \\
& $\%$ & $(1.0)$ & $(7.1)$ & $(25.8)$ & $(30.1)$ & $(17.5)$ \\
Antidepressant used & $\mathrm{N}$ & 0 & 73 & 56 & 6 & 40 \\
& $\%$ & 0 & $(15.2)$ & $(18.8)$ & $(2.6)$ & $(23.4)$ \\
Anxiolytics used & $\mathrm{N}$ & 39 & 151 & 166 & 73 & 53 \\
& $\%$ & $(39.4)$ & $(31.5)$ & $(55.7)$ & $(31.9)$ & $(31.0)$ \\
Hypnotics used & $\mathrm{N}$ & 4 & 7 & 21 & 140 & 10 \\
Anti-Pparkinsonian & $\%$ & $(4.0)$ & $(1.5)$ & $(7.0)$ & $(61.1)$ & $(5.8)$ \\
drug used & $\%$ & 87 & 180 & 207 & 90 & 69 \\
\hline & & $(87.9)$ & $(37.6)$ & $(69.5)$ & $(39.3)$ & $(40.4)$ \\
\hline
\end{tabular}

One polypharmacy study conducted in India found the similar results to our study, it showed that $31 \%$ of patients receive a combination of both FGAs and SGAs [16]. The rate of antipsychotic polypharmacy in India in our study was $25.7 \%$. These figures in India by two surveys are similar. The polypharmacy rate of Pakistan (51.7\%) was much higher than that of India (25.7\%). A number of reasons could be considered.

Mental health resources are much more limited in Pakistan compared to India. This shortcoming has contributed to the fragmentation of the mental health service system in Pakistan.

A fragmented health care system also contributes to polypharmacy. Patients are often treated by many clinicians who have their own individual prescription patterns. Due to a lack of adequate communication regarding a patient's care, the patients end up taking many drugs. North American and European countries have thoroughly worked on the availability of psychotropic drug prescriptions, however, limited work has been done on this topic in the Asian countries [17], [18]. Polypharmacy has frequently been seen in patients with complicated mental conditions. Patients who stay in psychiatric hospital for long periods usually suffer from severe health conditions or do not respond well to treatment, making them high risk for receiving polypharmacy [19], [20]. The latest studies have shown that patients with polypharmacy tend to stay hospitalized for longer periods of time than those who do not receive polypharmacy [21], [22].

For our survey, many chronic patients were enrolled. More than $60 \%$ of these 
patients had an illness duration of more than 5 years. It is expected that only difficult patients with severe psychotic symptoms consult with mental health services in countries with very limited psychiatric resources such as Pakistan.

The results of our survey also showed a high rate of combined medication with mood stabilizers in Pakistan (25.8\%) as compared to that in Bangladesh (1.0\%) and in India (7.1\%).

An 8-year follow up study conducted on schizophrenic patients in Pakistan showed that the rates of co-prescribed mood-stabilizers (carbamazepine) and antidepressants (fluoxetine) in patients receiving antipsychotic drugs are $38 \%$ and $23 \%$, respectively [4]. Those drugs are used for patients with severe emotional and mood problems. There are multiple reasons for the high rates of combined usage of mood stabilizers in Pakistan and other South Asian countries such as the presence of aggression or irritability, tenacious positive symptoms like delusions, and disorganized speech or as augmentation to antipsychotics that are either poorly tolerated or ineffective [23].

The current study was conducted to assess the psychotropic drug prescriptions patterns for patients with schizophrenia in Pakistan with comparable patterns among 15 countries in Asia. The findings showed that the use of anxiolytics in Pakistan was the highest (55.7\%) comparing to all other countries, Bangladesh (39.4\%), India (31.5\%), Japan (31.9\%, and Singapore (31.0) (Table 5). The total benzodiazepine use in patients in Pakistan was $67.7 \%(55.7 \%+7.0 \%)$ (Table 5), if "anxiolytics" and "hypnotics and sedatives" were added together (Table 5). A recent publication on psychotropic drugs in patients with schizophreniashowed that exposure to benzodiazepines has a dose-response relationship with mortality hazard ratios up to 1.74 [24]. In other words, patients with a chronic high dose usage of benzodiazepine have been associated with a higher risk of death as compared to those without exposure to benzodiazepines. There may be multiple reasons for the frequent use of benzodiazepine. One of the main reasons for the use of anxiolytics in Pakistan could be the absence of regulations regarding getting benzodiazepines over the counter. Further, general practitioners prescribe benzodiazepines to relieve physical symptoms in the presence of co-morbidities such as hypertension, and thyroid function problems. The lack of education regarding the side effects that results from the use of anxiolytics should be considered. Doctors in Pakistan resort to anxiolytics to reduce anxiety and insomnia, and to augment the effectiveness of the antipsychotic drugs. Anxiety is often seen as a symptom of major psychiatric illnesses, hence the co-prescription of anxiolytic drugs are often seen in treating anxiety as a symptom. We strongly suggest that physicians should be educated about the side effects of benzodiazepine to reduce the rate of use.

As previously mentioned, the use of typical antipsychotic drugs is about $15 \%$ which is much less when compared to the $31.6 \%$ use of atypical antipsychotics which has less tendency to cause antipsychotic induced Parkinsonian symptoms.

The co-prescription of anticholinergic medications is common in Asia for pa- 
tients with schizophrenia of all ages [25]

The rate of co-prescription of anti-Parkinsonian drugs is high (69.5\%) in $\mathrm{Pa}$ kistan. On the other hand, the rate of the usage of FGA was modest $(32,6)$. This is in sharp contrast with India (37.6\%), Japan (39.3\%) and Singapore (40.6\%). The rate of co-prescription of anti-Parkinsonian drugs has been decreasing with the decreased use of FGAs. It is unlikely that patients in Pakistan have strong sensitivities towards developing extrapyramidal symptoms. The only reason could be attributed to a lack of education and training on the proper use of anti-Parkinsonian drugs.

The above findings show the needs for further training and education in the proper prescription of psychotropic drugs in Pakistan.

This study has a limitation. Data were collected from only three major psychiatric centers in Pakistan: Karachi, Lahore, and Islamabad. The prescription pattern of those three centers may not represent the prescription trends of psychiatric facilities in other communities and in rural areas.

The main finding of the study was that majority of the patients were prescribed antipsychotic polypharmacy drugs in Pakistan. The possible reasons for the high rate of polypharmacy and high rate of co-prescription with anxiolytics, antidepressants and anti-Parkinsonian drugs have been discussed.

Past REAP surveys revealed that the prescription patterns of psychotropic drugs in a country are influenced by many factors. The scientific value of a drug plays a rather limited rôle. The national mental health delivery system, financing of drugs, insurance policies, local traditions, and even the history of a country influence on the prescription patterns [1], [2].

Psychotropic medication dispensation differs greatly across the world and in different time periods [13]. Many factors including illness identification, management plans, health belief models, access to treatment modalities, and the gravity of stigma are different in countries across the globe. Further education and training on the proper use of psychotropic drugs is recommended for psychiatrists in Pakistan. Guidelines on pharmacotherapy for patients with schizophrenia should be further developed and promoted.

\section{Acknowledgements}

This study was supported in part by the Taiwan Bureau of National Health Insurance (DOH92-NH-1025), Chang Gung Memorial Hospital (CMRPG83043) and the Taipei City Government (97001-62-010) in Taiwan. We express our thanks to all members who participated in REAP survey and in particular to those in Pakistan. Their names and affiliations are mentioned at the REAP home page (http://www.REAP.Asia).

\section{Disclosure}

All authors declare no potential conflicts of interest to report in relation to the contents of the study. 


\section{Author Contributions}

$\mathrm{MM}$, the first author, coordinated the project in Pakistan, and prepared the first draft of the manuscript. MUG, NA, NM, AJ, MMH and NSK collected data. SUY, LYC, SKL did the data analysis. NS, CHT, MYC and NS organized the whole survey. MS, SG and WWS made critical revision to the draft. All authors approved this submitted manuscript.

\section{References}

[1] Sim, K., Su, A., Fujii, S., et al. (2004) Antipsychotic Polypharmacy in Patients with Schizophrenia: A Multicentre Comparative Study in East Asia. British Journal of Clinical Pharmacology, 58, 178-183. https://doi.org/10.1111/j.1365-2125.2004.02102.x

[2] Chong, M.Y., Tan, C.H., Fujii, S., et al. (2004) Antipsychotic Drug Prescription for Schizophrenia in East Asia: Rationale for Change. Psychiatry and Clinical Neurosciences, 58, 61-67. https://doi.org/10.1111/j.1440-1819.2004.01194.x

[3] Jooma, R., Minhas, F.A. and Saxena, S. (2009) A Report of the Assessment of the Mental Health System in Pakistan Using the World Health Organization-Assesment Instrument for Mental Health Systems. http://www.who.int/mental_health/pakistan_who_aims_report.pdf

[4] Zahid, S., Durrani, F., Khan, R., Khalil, K. and Nazar, Z. (2016) Frequency of Psychotropic Drugs Use by Patientts with Schizophrenia in Eight Years Follow-Up. Journal of Postgraduate Medical Institute, 30, 48-51.

[5] Kane, J.M. and Correll, C.U. (2010) Past and Present Progress in the Pharmacologic Treatment of Schizophrenia. Journal of Clinical Psychiatry, 71, 1115-1124. https://doi.org/10.4088/JCP.10r06264yel

[6] Davis, J.M. (1975) Overview: Maintenance Therapy in Psychiatry: I. Schizophrenia. American Journal of Psychiatry, 132, 1237-1245. https://doi.org/10.1176/ajp.132.12.1237

[7] Correll, C.U. and Gallego, J.A. (2012) Antipsychotic Polypharmacy: A Comprehensive Evaluation of Relevant Correlates of a Long-Standing Clinical Practice. Psychiatric Clinics of North America, 35, 661-681. https://doi.org/10.1016/j.psc.2012.06.007

[8] Balter, M.B., Levine, J. and Manheimer, D.I. (1974) Cross-National Study of the Extent of Anti-Anxiety-Sedative Drug Use. The New England Journal of Medicine, 290, 769-774. https://doi.org/10.1056/NEJM197404042901404

[9] Kapczinski, F., Amaral, O.B., Madruga, M., Quevedo, J., Busnello, J.V. and de Lima, M.S. (2001) Use and Misuse of Benzodiazepines in Brazil: A Review. Substance Use \& Misuse, 36, 1053-1069. https://doi.org/10.1081/JA-100104489

[10] Iqbal, S.P., Ahmer, S., Farooq, S., et al. (2011) Benzodiazepine Use among Adults Residing in the Urban Settlements of Karachi, Pakistan: A Cross Sectional Study. Substance Abuse Treatment, Prevention, and Policy, 6, 19. https://doi.org/10.1186/1747-597X-6-19

[11] Xiang, Y.T., Ungvari, G.S., Correll, C.U., Chiu, H.F. and Shinfuku, N. (2016) Trends in the Access to and the Use of Antipsychotic Medications and Psychotropic Co-Treatments in Asian Patients with Schizophrenia. Epidemiology and Psychiatric Sciences, 25, 9-17. https://doi.org/10.1017/S2045796015000694

[12] Bowers, L., Callaghan, P., Clark, N. and Evers, C. (2004) Comparisons of Psychotropic Drug Prescribing Patterns in Acute Psychiatric Wards across Europe. Euro- 
pean Journal of Clinical Pharmacology, 60, 29-35. https://doi.org/10.1007/s00228-003-0719-7

[13] Rothbard, A.B., Kuno, E. and Foley, K. (2003) Trends in the Rate and Type of Antipsychotic Medications Prescribed to Persons with Schizophrenia. Schizophrenia Bulletin, 29, 531-540. https://doi.org/10.1093/oxfordjournals.schbul.a007025

[14] Fatima, A., Barkat, U., Mahmood, K. and Zaka, M. (2011) Schizophrenia in Elderly Patients. Journal of Pharmaceutical Sciences and Research, 3, 952-960.

[15] Kukreja, S., Kalra, G., Shah, N. and Shrivastava, A. (2013) Polypharmacy in Psychiatry: A Review. Mens Sana Monographs, 11, 82-99. https://doi.org/10.4103/0973-1229.104497

[16] Ramadas, S., Kuttichira, P., Sumesh, T.P. and Ummer, S.A. (2010) A Study of an Antipsychotic Prescription Pattern of Patients with Schizophrenia in a Developing Country. Indian Journal of Psychological Medicine, 32, 13-16. https://doi.org/10.4103/0253-7176.70520

[17] Gallego, J.A., Bonetti, J., Zhang, J., Kane, J.M. and Correll, C.U. (2012) Prevalence and Correlates of Antipsychotic Polypharmacy: A Systematic Review and Meta-Regression of Global and Regional Trends from the 1970s to 2009. Schizophrenia Research, 138, 18-28. https://doi.org/10.1016/j.schres.2012.03.018

[18] Huskamp, H.A. (2005) Pharmaceutical Cost Management and Access to Psychotropic Drugs: The U.S. Context. International Journal of Law and Psychiatry, 28, 484-495. https://doi.org/10.1016/j.ijlp.2005.08.004

[19] Kreyenbuhl, J., Marcus, S.C., West, J.C., Wilk, J. and Olfson, M. (2007) Adding or Switching Antipsychotic Medications in Treatment-Refractory Schizophrenia. Psychiatric Services, 58, 983-990. https://doi.org/10.1176/ps.2007.58.7.983

[20] Santone, G., Bellantuono, C., Rucci, P., Picardi, A., Preti, A. and De Girolamo, G. (2011) Patient Characteristics and Process Factors Associated with Antipsychotic Polypharmacy in a Nationwide Sample of Psychiatric Inpatients in Italy. Pharmacoepidemiology and Drug Safety, 20, 441-449. https://doi.org/10.1002/pds.2083

[21] Ghio, L., Natta, W., Gotelli, S. and Ferrannini, L. (2011) Antipsychotic Utilisation and Polypharmacy in Italian Residential Facilities: A Survey. Epidemiology and Psychiatric Sciences, 20, 171-179. https://doi.org/10.1017/S2045796011000242

[22] Centorrino, F., Goren, J.L., Hennen, J., Salvatore, P., Kelleher, J.P. and Baldessarini, R.J. (2004) Multiple versus Single Antipsychotic Agents for Hospitalized Psychiatric Patients: Case-Control Study of Risks versus Benefits. American Journal of Psychiatry, 161, 700-706. https://doi.org/10.1176/appi.ajp.161.4.700

[23] Sim, K., Yong, K.H., Chan, Y.H., et al. (2011) Adjunctive Mood Stabilizer Treatment for Hospitalized Schizophrenia Patients: Asia Psychotropic Prescription Study (2001-2008). International Journal of Neuropsychopharmacology, 14, 1157-1164. https://doi.org/10.1017/S1461145711000563

[24] Tiihonen, J., Mittendorfer-Rutz, E., Torniainen, M., Alexanderson, K. and Tanskanen, A. (2016) Mortality and Cumulative Exposure to Antipsychotics, Antidepressants, and Benzodiazepines in Patients with Schizophrenia: An Observational Follow-Up Study. American Journal of Psychiatry, 173, 600-606.

https://doi.org/10.1176/appi.ajp.2015.15050618

[25] Xiang, Y.T., Dickerson, F., Kreyenbuhl, J., et al. (2013) Common Use of Anticholinergic Medications in Older Patients with Schizophrenia: Findings of the Research on Asian Psychotropic Prescription Pattern (REAP) Study, 2001-2009. International Journal of Geriatric Psychiatry, 28, 305-311. https://doi.org/10.1002/gps.3827 